\title{
Optimization Algorithm Based on Laplacian Location and Extension Grid
}

\author{
Shaohui $\mathrm{Ma}^{1}$, Xinling Sun ${ }^{1}$, Benzhai $\mathrm{Hai}^{2}$ and Xie Rui-yun \\ ${ }^{1}$ Department of Computer Science and Technolog, Henan Institute of Technology, \\ Henan Xinxiang 453000, China \\ ${ }^{2}$ College of Computer \& Information Engineering, Henan Normal University, \\ Xinxiang, Henan, 453007, China \\ 924327262@qq.com
}

\begin{abstract}
This article put forward an optimization algorithm of triangle grid model based on Laplacian coordinate constraint and a fast reconstruction algorithm of model topology structure based on STL file, such methods may enhance the quality of triangular patch as well as splendidly remain local geometric features of original grid model. The character of it is there's unnecessary to distinguish constraint vertex and free vertex no longer and to carry on a double restraints to location and Laplacian coordinate for all vertex, then solve a linear system, which contains double restraint, with least-squares sense to carry on an relocation for all vertex. The experimental results showed that proposed algorithm is provided with a certain advantage in terms of detail features of grid compared with precedent Laplacian optimization algorithm.
\end{abstract}

Keywords: Triangular grid model; Discrete differential geometry; Surface; Global optimization

\section{Introduction}

As an enhancement of software function, there have been a number of measuring equipment may output measured data and triangular grid data (namely data after being trianglized) or STL (Stereolithography) format data for the time being. Among it a series of small triangular planes are adopted in STL file format, which now has become one of industrial standards of file format with CAD/CAM system interface, to draw near solid model. The quality will be generally inferior if directly use grid model described by STL file, which is generated from measurement of measuring equipment, moreover, there will appear to be issues like serious asymmetry of triangular planes (an excess of long-narrow and tabular triangular planes). An optimization shall be taken to such models in order to boost the efficiency and quality of subsequent disposal process for reverse engineering. The purpose of triangular grid optimization is to advance the quality of grid surface as much as possible on the premise that how to control geometric distortion of of grid surface within certain limits, methods including optimization algorithm of altering and maintaining connected relation of grid, a global optimization algorithm without altering connected relation of grid is mainstream research for the time being. Such algorithm in this article that put forward is based on all kinds of optimization algorithms above with comprehensive research, the character of it is there's unnecessary to distinguish constraint vertex and free vertex no longer and to carry on a double restraints to location and Laplacian coordinate for all vertex, then solve a linear system, which contains double restraint, with least-squares sense to carry on an relocation for all vertex. The virtue of proposed algorithm is it's provided with favorable retainability to local geometric features of model. 


\section{Evaluation Criterion of Grid Quality}

If to carry on an optimization to grid model, for one thing, an evaluation criterion quantized of grid quality shall be provided, this article put to use an regular degree of side length to estimate the quality of single triangular patch. Computing method is shown as formula (1).

$$
r_{e}=\frac{l_{3}-l_{1}}{l_{2}}
$$

Where, $r_{e}$ is regular degree of triangle based on side length, $l_{i}$ is one side of triangle, $l_{1}<l_{2}<l_{3}, O \leq r_{e}<1$, when triangular patch is an regular triangle, $r_{e}=0$, when is a long-narrow triangular mesh, $r_{e}$ is close to 1 . If most of regular degrees of triangular patch in a model are close to 0 , which means that such model is provided with great quality, the other way around, the quality of such model will be described with mass diagram.

\section{Reconstruction Algorithm of Grid Surface Model}

\subsection{Algorithm Procedure}

The optimization algorithm of model quality in this article is divided into three steps, as described below:

Step 1: reconstruct topological structure of model in STL file.

Step 2: compute two types of Laplacian coordinate of grid model vertex.

Step 3: create a linear system of double constraints which includes vertex location and Laplacian coordinate, then carry out an relocation by solving least square solution.

Compared with solid model, a mesh surface is normally integrated with tens of thousands or even hundreds of thousands of triangular patches, which brings about many a STL file data size, and the coordinate information of vertex may have an repeated emergence in multi-triangular patches, therefore, a great deal of research work has been done on how to chip away redundant data and establish efficiently reasonable topological structure by a lot of researchers at abroad [9, 10, 11, 12 and 13]. Based on an inherent correlation in STL file data, this article put forward a fast reconstruction algorithm, which requires no complex data structure, based on various reconstruction algorithms, which may realize an reconstruction of topological structure in case of giving less amount of calculated quantities.

As described below:

Step 1: establish vertex array Vertex $\left(3,^{*}\right)$ and topology array topology $\left(3,{ }^{*}\right)$.

Step 2: load in turn the data of each triangular patch (let it be triangular patch j) in STL file, then write vertex coordinate into vertex as serial numbering clockwise, and write vertex code into topology in the same order.

Step 3: compare with each vertex coordinate in patch of $\mathrm{j}$ code and three vertex coordinates in patch of $\mathrm{j}-1$ code of previous patch; if the $1(\mathrm{i}=1,2,3)$ vertex coordinate in patch of $\mathrm{j}$ code equals to the $\mathrm{k}(\mathrm{k}=1,2,3)$ vertex coordinate in patch of $\mathrm{j}-1$ code, then topology $(\mathrm{i}, \mathrm{j})=$ topology $(\mathrm{k}, \mathrm{j}-1)$; otherwise carry out step 4 .

Step 4: make an reverse comparison of the $i(i=1,2,3)$ vertex coordinate in patch of $j$ code and vertex array $(3, n)$ (suppose that it has generated $n$ vertex array); if coordinate value of the code of $m(m \leq n)$ existed is identical to it, then topology $(i, j)=m$, otherwise it's 
a new vertex, let $n=n+1$, and write the $i(i=1,2,3)$ vertex coordinate of the patch of $j$ code into $\operatorname{Vertex}(3, \mathrm{n})$.

Step 5: let $\mathrm{j}=\mathrm{j}+1$ after having all vertex in the patch of $\mathrm{j}$ code completed and return to Step 2.

\subsection{Laplacian coordinate of grid vertex}

Discrete Laplacian coordinate on grid is the most popular mathematical tool to describe geometric details, Leplacian coordinate is employed in many a optimization algorithm of mesh model. After having Step 2 completed, vertex array Vertex and triangular patch topology both are obtained, then compute two types of Laplacian of mesh vertex according to these two array data structures, computational formula as follows:

$$
\delta_{i}=\sum_{\mathrm{v}_{j} \in \mathrm{N}(\mathrm{i})} w_{i j}\left(v_{j}-v_{i}\right)
$$

Formula (2) is the computational formula for Laplacian coordinate at vertex Vi of grid model, $\mathrm{Vj}$ is an random vertex in partial patch of vertex $\mathrm{Vi}$.

$$
w_{i j}=\frac{\omega_{i j}}{\sum_{v j \in N(i)} \omega_{i j}}
$$

Formula (3), (4) and (5) are computational formulas for weight coefficient, Laplacian coordinate, which computed from formula (4) and (5), can be called as identical Laplacian coordinate and cotangent Laplacian coordinate, which are recorded as $\delta_{u}$ and $\delta_{c}$, respectively.

$$
\begin{gathered}
\omega_{u i j}=1 \\
\omega_{i i j}=\cot \alpha_{i j}+\cot \beta_{i j}
\end{gathered}
$$

This article put to use of Laplacian matrix L of $n \times n$ while computing Laplacian coordinate of grid vertex.

$$
L=\left|\begin{array}{ccc}
L_{11} & \cdots & L_{1 n} \\
\vdots & L_{i j} & \vdots \\
L_{n 1} & \cdots & L_{n n}
\end{array}\right|
$$

The form of $L_{i j}$ in formula (6) is the same as formula (7).

$$
\begin{gathered}
L_{i j}=\left\{\begin{array}{cc}
-1 & i=j \\
w_{i j} & v_{j} \in N(i) \\
0 & \text { otherwise }
\end{array}\right. \\
\Delta_{d}=L V_{d}=\left[\delta_{1 d}, \delta_{2 d}, \delta_{2 d}, \cdots \delta_{n d}\right]^{T}, d \in\{x, y, z\} \\
V_{d}=\left[V_{d 1}, V_{d 2}, V_{d 3}, \cdots, V_{d n}\right]^{T}, d \in\{x, y, z\}
\end{gathered}
$$

$V_{d}$ in formula (9) is a weight of vertex coordinate at some coordination orientation, $\Delta_{d}$ in formula (8) is a weight of Laplacian coordinate at some coordinate orientation.

$\bar{k}_{i} n_{i}=\delta_{c i \bar{k}}=\frac{1}{4 A\left(v_{i}\right)} \sum_{v_{j \in N}(i)}\left(\cot \alpha_{i j}+\cot \beta_{i i}\right)\left(v_{i}-v_{j}\right)$

$\bar{k}_{i}$ in formula (10) is an average curvature of grid surface at vertex Vi. $A\left(v_{i}\right)$ is Voronoi figure (dash area in figure 1) of vertex Vi. 


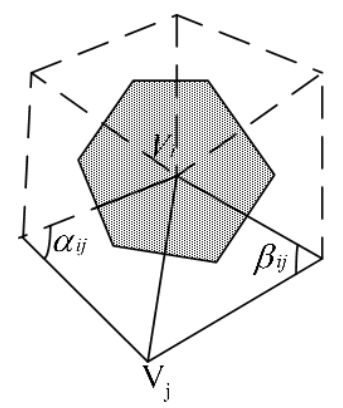

\section{Figure 1. Partial Patch of Vertex Vi}

\subsection{Relocation of Grid Vertex}

$V_{d}^{\prime}$ in formula (11) is a weight of vertex coordinate relocated at some coordinate orientation, optimization method in this article is to solve these three weights to grid vertex coordinate, what requires to be solved is a linear system of $2 n \times n$ indicated in formula (12).

$$
\begin{aligned}
& V_{d}^{\prime}=\left[V_{d 1}^{\prime}, V_{d 2}^{\prime}, V_{d 3}^{\prime}, \cdots, V_{d n}^{\prime}\right]^{T}, d \in\{x, y, z\} \\
& {\left[\frac{L_{u}}{W_{p}}\right] V_{d}^{\prime}=\left[\frac{\Delta_{d c}}{W_{p} V_{d}}\right]} \\
& \left\{\begin{array}{cc}
L_{u} V^{\prime} d=\Delta_{d c} & <1> \\
W_{p} V^{\prime} d=W_{p} V_{d} & <2>
\end{array}\right.
\end{aligned}
$$

$L_{u}$ in formula (12) is Laplacian matrix of identical Laplacian coordinate, $\Delta_{d c}$ is a weight of Laplacian coordinate, $W_{p}$ is constrained control matrix, that is, a $n \times n$ diagonal matrix. The linear system in formula (12) can be further written as the form of formula (13), formula (13) shows that the linear system in formula (12) is composed of geometric constraint system $\langle 1\rangle$ and position constraint system $<2\rangle$, each constraint system contains $n$ equations, the arbitrary coordinate weight of each vertex on grid is constrained by two constraint systems respectively. Nealen put forward that the thought of employing <1> in formula 13 to construct geometric constraint system in 2005 [14], the rationale is: when all triangular patches, which contained in partial patch of arbitrary vertex on grid surface, are isosceles triangles, the identical Laplacian coordinate and cotangent Laplacian on this vertex are equal to one another. When a certain triangular patch, according to above conclusion, is expended to the whole model surface, it can be found that: if all triangular patches are close to regular triangle, all identical Laplacian coordinates and cotangent Laplacian coordinates are close to each other.

The linear system required to be solved in this article is an incompatible overdetermined equation, as to such equation, we can only obtain the approximate solution of least-squares sense, the function of constraint control matrix $W_{p}$ is to adjust the influence of two types of constraint systems on optimization results, namely the influence on approximate solution obtained at last. When the setting of $W_{p}$ is partial to geometric constraint, the quality of optimization results grid will be better, however, the error between original grid will be more serious, when the setting of $W_{p}$ is partial to position constraint, the result will be reversed, therefore it's an important issue on how to set $W_{p}$ to make an optimized results strike a balance between grid quality and distortion factor. $w_{p i}$ in Formula (14) is the element of $W_{p}$ in i line as well as position constraint weight of vertex, the setting process of $W_{p}$ is the process to determine $W_{p i}$. 


$$
w_{p i}=\sum_{j=1}^{n\left(\overline{k_{i}}\right)} \frac{\bar{k}_{i}\left(\bar{k}_{i}-\bar{k}_{\min }\right)}{n\left(\bar{k}_{i}\right)}
$$

$\bar{k}_{i}$ in formula (14) is an average curvature ( $\overline{k_{i}}$ is scalar and mean curvature vector is $\left.\bar{k} n_{i}\right)$ of vertex $\mathrm{Vi}, \bar{k}_{\min }$ is all vertex's minimum of average curvature on grid.

\section{Experiments and Analysis}

Proposed algorithm is achieved by integrating OpenGL graphics library taking Visual $\mathrm{C}++2010$ as development platform, contrast test is carried out between PC, which CPU dominant frequency is 2.8 hertz and internal storage is $2 \mathrm{G}$, and optimization algorithm, which put forward by Nealen in 2015, based on characteristic vertex in many respects, the experimental result as follows: for one thing, bird model with 23,455 surface patches were used to conduct an experiment, then the experimental results were reviewed from memory consumption, algorithm execution time, quality of grid model with optimization and distortion factor of model. Figure 2 is denoted as original model and its grid, curve graph in figure 3 is mass curve of grid. Abscissa axis of mass curve is the regular degree of triangular patch, vertical axis is provided with quantity of triangular patch of some regular degree. It's important to note that the regular degree of each triangular patch is a real number between 0 and 1, the regular degree of two triangular patches whose are entirely identical are rare to be seen, in order to structure a visual mass curve, it's divided into 100 section from 0 to 1 , the regular degree which belongs to triangular patch of same section is regarded as being provided with identical regular degree and its value is the mid point of this section.

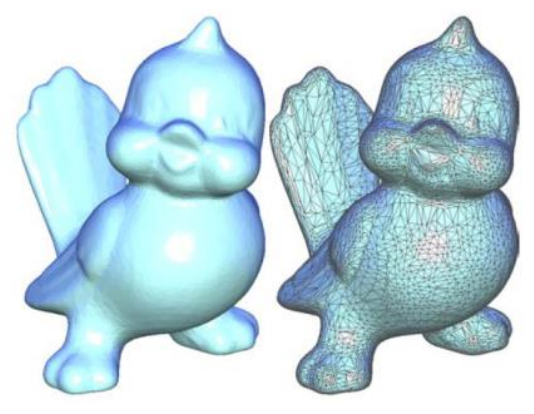

Figure 2. Representation of Original Bird Mode and its Grid

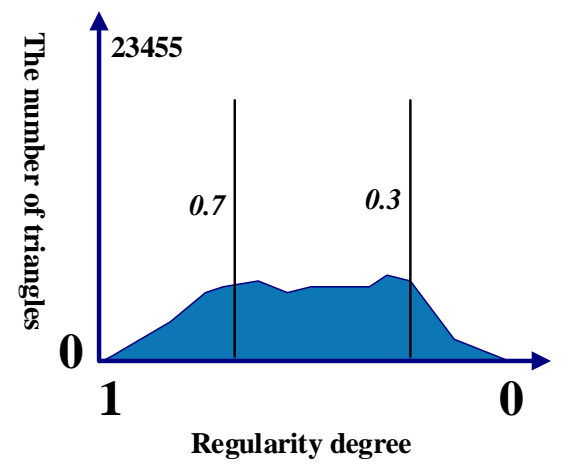

Figure 3. Grid Mass Curve of Original Bird Model

It's observed that the regular degree of triangular patch in original grid mostly centralize between 0.7 and 0.3 from the mass curve in Figure 3, and there has few high quality triangular patches. 


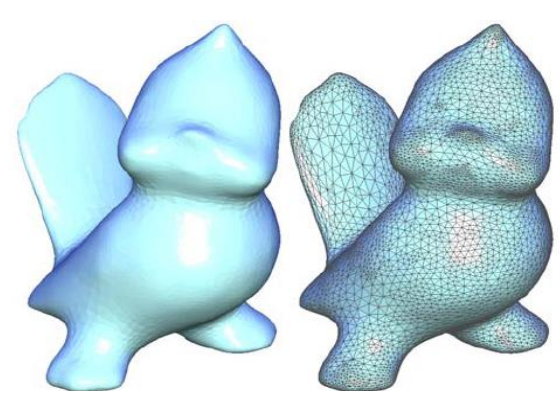

Figure 4. Representation of Bird Model and its Grid with Optimization

Figure 4 is the representation of bird model and its grid with optimization of Nealen algorithm, Figure 5 is the mass curve of model with optimization of Nealen algorithm. It's observed that the quality of grid is significantly improved on curve, most regular degrees of triangular patches are all centralized between 0.3 and 0.03 , however, it can be seen that geometric detail of grid surface is provided with significant distortion in Figure 4.

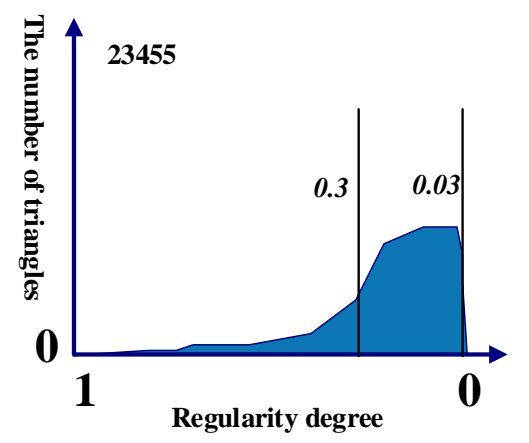

Figure 5. Mass Curve of Model with Nealen Algorithm Optimization

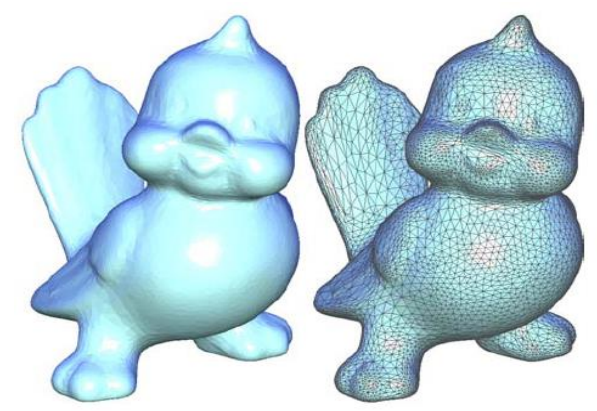

Figure 6. Representation of Bird Model and its Grid with Proposed Algorithm Optimization

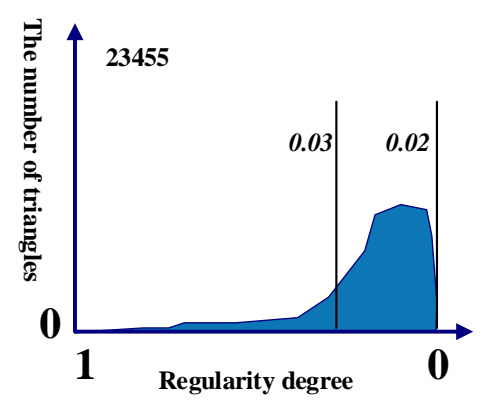

Figure 7. Representation of Bird Model and its Grid with Proposed Algorithm Optimization 
The model in Figure 7 is the representation of bird model and its grid with proposed algorithm optimization, and figure 7 is its corresponding mass curve. The quality of model with proposed algorithm optimization is slightly improved than the model with Nealen algorithm optimization, most of regular degrees of triangular patches are centralized between 0.02 and 0.3 , more importantly, figure 6 shows that proposed algorithm well preserve some geometric details of original model with lower distortion. Theoretically, it's analyzed that it is because Nealen algorithm has to determine the position of partial features remain unchanged, to meet up with this kind of rigid constraint, the algorithm has to sacrifice partial geometric details in peripheral region of feature vertex, proposed algorithm however is to relocate to all vertex on model, although there has no few vertex to maintain its position unchanged, it keeps up geometric details of model in a better way as a whole.

\section{Conclusion}

This article put forward an optimization algorithm of triangle grid model based on Laplacian coordinate constraint and a fast reconstruction algorithm of model topology structure based on STL file, the purpose of carrying out a global optimization for triangular grid model of file format of STL file can be achieved by combing these two algorithms. A contrast test was conducted between proposed algorithm and Nealen algorithm, the reason why Nealen algorithm is selected to conduct a contrast test is because such algorithm is for the being rather effective as well as representative optimization algorithm of surface grid model, some new algorithms that put forward in recent years mostly are related to the improvement and development of such algorithm. The experimental result at last showed that proposed algorithm significantly enhanced the quality of grid model as well maintained well geometric details of it, the distortion factor of model with optimization is lower than Nealen algorithm, at the same time, formula (12) also provided an open algorithm platform in which a targeted optimization effect can be gained by selecting different ${ }^{W_{p}}$ designing scheme.

\section{Acknowledgment}

The research is supported by National Natural Science Foundation of China (No.U1404602) key scientific research project of Henan province (No.15B520006); Henan Provincial Department of Education Science and technology research key project (14A520046); key scientific research project of Henan province (15A520063).

\section{References}

[1] Guo C, Liu X, Jin M. The research on optimization of auto supply chain network robust model under macroeconomic fluctuations[J]. Chaos, Solitons \& Fractals, (2015).

[2] $\mathrm{Li} \mathrm{X}, \mathrm{Lv} \mathrm{Z}, \mathrm{Hu}$ J. XEarth: A 3D GIS Platform for managing massive city information[C]//Computational Intelligence and Virtual Environments for Measurement Systems and Applications (CIVEMSA), 2015 IEEE International Conference on. IEEE, (2015): 1-6.

[3] Yang J, Chen B, Zhou J. A Low-Power and Portable Biomedical Device for Respiratory Monitoring with a Stable Power Source[J]. Sensors, (2015), 15(8): 19618-19632.

[4] Guanqun Bao, Liang Mi, Yishuang Geng, Kaveh Pahlavan, A computer vision based speed estimation technique for localiz ing the wireless capsule endoscope inside small intestine, 36th Annual International Conference of the IEEE Engineering in Medicine and Biology Society (EMBC), Aug. (2014).

[5] Xinchao Song, Yishuang Geng, Distributed community detection optimization algorithm for complex networks, Journal of Networks, 9(10), 2758-2765, Jan. (2014).

[6] Jiang D, Ying X, Han Y. Collaborative multi-hop routing in cognitive wireless networks[J]. Wireless Personal Communications, (2015): 1-23.

[7] Jinyu Hu and Zhiwei Gao. Modules identification in gene positive networks of hepatocellular carcinoma using Pearson agglomerative method and Pearson cohesion coupling modularity[J]. Journal of Applied Mathematics, 2012 (2012). 
[8] Jiang $\mathrm{D}, \mathrm{Xu} \mathrm{Z}$, Chen Z. Joint time-frequency sparse estimation of large-scale network traffic[J]. Computer Networks, 2011, 55(15): 3533-3547.Jinyu Hu, Zhiwei Gao and Weisen Pan. Multiangle Social Network Recommendation Algorithms and Similarity Network Evaluation[J]. Journal of Applied Mathematics, 2013 (2013).

[9] Mingda Zhou, Guanqun Bao, Yishuang Geng, Bader Alkandari, Xiaoxi Li, Polyp detection and radius measurement in small intestine using video capsule endoscopy, 2014 7th International Conference on Biomedical Engineering and Informatics (BMEI), Oct. (2014).

[10] Gan Yan, Yuxiang Lv, Qiyin Wang, Yishuang Geng, Routing algorithm based on delay rate in wireless cognitive radio network, Journal of Networks, 9(4), 948-955, Jan. (2014).

[11] Lin Y, Yang J, Lv Z. A Self-Assessment Stereo Capture Model Applicable to the Internet of Things[J]. Sensors, (2015) 15(8): 20925-20944.

[12] Wang K, Zhou X, Li T. Optimizing load balancing and data-locality with data-aware scheduling[C]. Big Data (Big Data), 2014 IEEE International Conference on. IEEE, (2014): 119-128.

[13] Zhang L, He B, Sun J. Double Image Multi-Encryption Algorithm Based on Fractional Chaotic Time Series[J]. Journal of Computational and Theoretical Nanoscience, (2015), 12: 1-7.

[14] Su T, Lv Z, Gao S. 3d seabed: 3d modeling and visualization platform for the seabed[C]. Multimedia and Expo Workshops (ICMEW), 2014 IEEE International Conference on. IEEE, (2014): 1-6.

[15] Shuang Zhou, Liang Mi, Hao Chen, Yishuang Geng, Building detection in Digital surface model, 2013 IEEE International Conference on Imaging Systems and Techniques (IST), Oct. (2012).

[16] Jie He, Yishuang Geng, Kaveh Pahlavan, Toward Accurate Human Tracking: Modeling Time-of-Arrival for Wireless Wearable Sensors in Multipath Environment, IEEE Sensor Journal, 14(11), 3996-4006, Nov. (2014).

[17] Lv Z, Halawani A, Fen S. Touch-less Interactive Augmented Reality Game on Vision Based Wearable Device[J]. Personal and Ubiquitous Computing, (2015), 19(3): 551-567.

[18] Guanqun Bao, Liang Mi, Yishuang Geng, Mingda Zhou, Kaveh Pahlavan, A video-based speed estimation technique for localizing the wireless capsule endoscope inside gastrointestinal tract, 2014 36th Annual International Conference of the IEEE Engineering in Medicine and Biology Society (EMBC), Aug. (2014).

[19] Degui Zeng, Yishuang Geng, Content distribution mechanism in mobile P2P network, Journal of Networks, 9(5), 1229-1236, Jan. (2014).

[20] Gu W, Lv Z, Hao M. Change detection method for remote sensing images based on an improved Markov random field[J]. Multimedia Tools and Applications, (2015): 1-16.

[21] Chen Z, Huang W, Lv Z. Towards a face recognition method based on uncorrelated discriminant sparse preserving projection[J]. Multimedia Tools and Applications, (2015): 1-15.

[22] Jinyu Hu and Zhiwei Gao. Distinction immune genes of hepatitis-induced heptatocellular carcinoma[J]. Bioinformatics, (2012), 28(24): 3191-3194.

[23] Su T, Wang W, Lv Z, et al. Rapid Delaunay triangulation for randomly distributed point cloud data using adaptive Hilbert curve[J]. Computers \& Graphics, (2016), 54: 65-74.

\section{Authors}

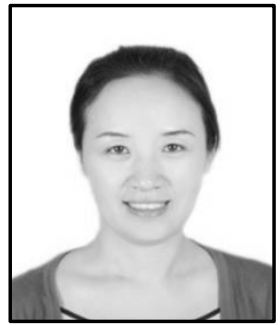

Ma Shaohui, received her M.S. degree in Electronic and Information Engineering from Xi'an Jiaotong university in Xi'an. She is currently an associate professor in Henan Institute of Technology. Her research interest is mainly in the area of Computer Software, Mechanical and Electrical Integration. She has published several research papers in scholarly journals in the above research areas 